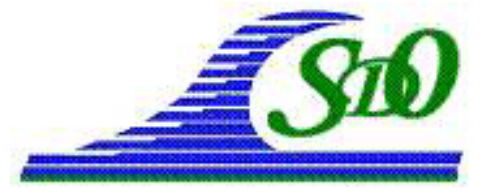

XI ìmes Journées Nationales Génie Côtier - Génie Civil

Les Sables d'Olonne, 22-25 juin 2010

DOI:10.5150/jngcgc.2010.002-A C Editions Paralia CFL

disponible en ligne - http://www.paralia.fr - available online

\title{
Modélisation des états de mer du globe à la plage : validation de nouveaux paramètres produits par Prévimer
}

\author{
Fabrice ARDHUIN $^{1}$, Rudy MAGNE ${ }^{2}$
}

1. Ifremer, Laboratoire d'Océanographie Spatiale, BP 70, 29280 Plouzané, France. ardhuin@ifremer.fr

2. SHOM, division HOM, 13 rue du Chatellier, 29228 BREST CEDEX 2, France. rudy.magne@shom.fr

\section{Résumé :}

Un système de prévision des vagues a été mis en place par le SHOM dans le cadre du projet inter-organismes "Prévimer" (http://www.previmer.org). Les applications visées sont multiples, depuis l'information du grand public jusqu'aux applications scientifiques et techniques, y compris la télédétection, et le génie côtier. En 2009, les principales améliorations ont porté sur la mise en service de nouveaux domaines de calcul traités avec le code de calcul WAVEWATCH III $^{\mathrm{TM}}$ dans sa version à maillage régulier ou irrégulier, avec des résolutions atteignant $150 \mathrm{~m}$ à la côte. Les courants en Manche et mer d'Iroise sont désormais pris en compte et les modèles fournissent de nouveaux paramètres: dérive à la surface induite par les vagues, tensions de radiation... Des conditions aux frontières sont aussi fournies pour d'autres activités de prévision. La validation des modèles à toutes ces échelles continue de faire l'objet d'efforts particuliers alliant télédétection spatiale et campagnes de mesure in situ. Ainsi, à la position de la bouée "Pierres Noires" les erreurs de prévision ont pu être réduite de 17\% pour la hauteur significative $\mathrm{H}_{\mathrm{m} 0}$ à environ $10 \%$, essentiellement du fait de la prise en compte des courants. Par ailleurs, l'indice de hauteur d'eau $\left(\mathrm{g} \mathrm{H}_{\mathrm{m} 0}\right)^{0.5} \mathrm{~T}_{\mathrm{m} 0,-1}$ issu des modèles est fortement corrélé ( $\mathrm{r}=0.94$ ) aux mesures de maxima de niveau dans l'archipel de Molène ainsi qu'aux amplitudes de seiche enregistrées à Port Tudy (Ile de Groix). La généralité de ce résultat reste à établir par des campagnes de mesure sur d'autres sites, mais il permet déjà un prévision du risque local de submersion, comme cela a été le cas pour l'île de Bannec (archipel de Molène) lors de la tempête de mars 2008. Les développements actuels du système visent à améliorer encore la prévision et permettre le calcul de nouveau paramètres tels que les statistiques de déferlement.

\section{Mots-clés :}

Modélisation des états de mer - Prévimer - Projet IOWAGA - Courant - Seiche

\section{Introduction}

Le système de prévision des vagues mis en place par le SHOM dans le cadre du projet inter-organismes "Prévimer" (http://www.previmer.org), a déjà été décrit par MAGNE et ARDHUIN (2008). Des évolutions importantes ont eu lieu en 2009 et se poursuivent, 
avec le soutien du Conseil Européen de la Recherche avec le projet "Integrated Ocean waves for Geophysical and other Applications" (IOWAGA). Il s'agit en particulier de proposer des produits mieux adaptés à différentes applications, depuis l'information du grand public jusqu'aux applications scientifiques et techniques, dont la télédétection et le génie côtier. Le présent article vise donc à détailler ces évolutions en insistant ensuite sur la validation quantitative des différents paramètres estimés.

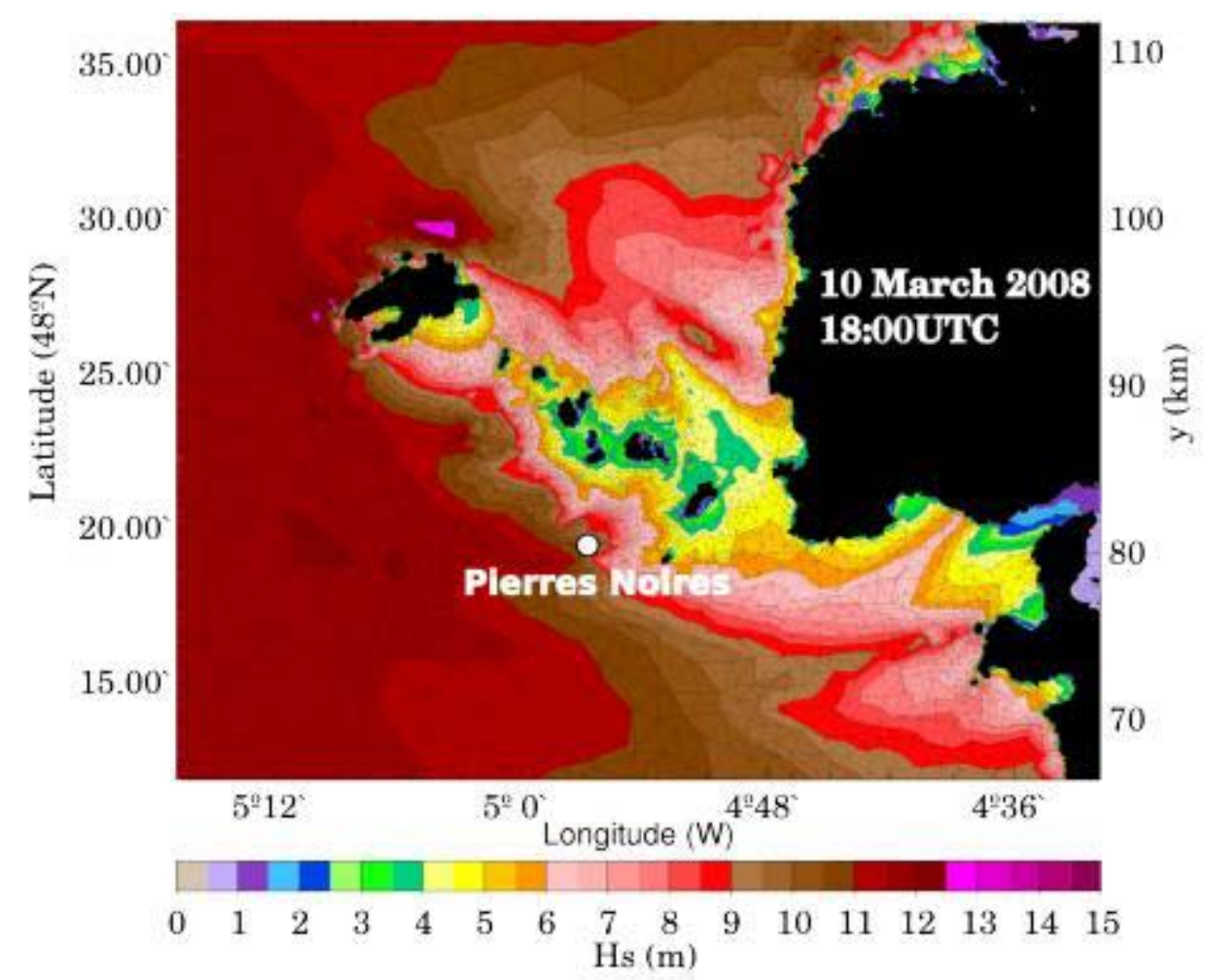

Figure 1. Exemple de carte de hauteur significative calculée sur la côte du Finistère nord, le soir de la tempête du 10 mars 2008. Le maillage, utilisé pour Prévimer, résoud les îles de l'archipel de Molène. L'île de Banneg, largement submergée à ce moment là, est la première île au sud-est d'Ouessant. Le modèle utilise les courants et niveaux d'eau des autres modèles Prévimer, mais il n'est pas capabable, à l'heure actuelle, de représenter cette submersion, du fait des très petites échelles spatiales nécessaires avec la technique employée ici (20 m ou moins), et de l'absence de couplage vagues-surcote.

\section{Evolutions du système en 2009}

En 2009, les principales améliorations ont porté sur

- la mise en service de nouveaux domaines de calcul traités avec le code de calcul WAVEWATCH III ${ }^{\mathrm{TM}}$ (ci dessous appelé WWATCH) dans sa version à maillage régulier (Littoral méditerranéen français - y compris la Corse, Nouvelle Calédonie, Polynésie Française), ou irrégulier de type "éléments finis" (Manche Occidentale et 
mer d'Iroise), avec des résolutions atteignant $150 \mathrm{~m}$ à la côte, comme l'illustre la figure 1. Ces domaines s'ajoutent aux domaines existants (Océan Global, Plateau nord-ouest Européen, Gascogne-Manche-Mer du Nord, Antilles françaises),

- la prise en compte des courants en Manche et mer d'Iroise

- l'estimation de nouveaux paramètres: dérive à la surface, tensions de radiation, ... listés dans le tableau 1. Ces paramètres sont généralement fournis via ftp à l'adresse http://www.tinyurl.com/yetsofy dans les 2 heures suivant la date du calcul, avec une échéance de prévision jusqu'à 6 jours. Il s'agit de rejeux ("hindcast") mais aussi des prévisions.

- la fourniture de conditions aux frontières (spectresà pour d'autres activités de prévision (par exemple: projet LOREA). Actuellement les positions des points de sorties pour ces spectres sont arrondies au 1/10ème de degré le plus proche en latitude et en longitude, afin de limiter la quantité d'information quand plusieurs zones de zoom se chevauchent.

Des visualisations des principaux paramètres sont disponibles sur le site web :

http://www.previmer.org/previsions/vagues/ sous la forme de cartes, de séries temporelles, et de spectres directionnels pour quelques points pré-sélectionnés. Par ailleurs les données numériques de l'ensemble des spectres sortis par le modèle sont sur le site ftp, avec aussi un résulmé sous forme de "bulletin texte", donnant les différents systèmes de houle. Par exemple, pour la bouée OMM 62163 "Brittany", le fichier mww3.62163.split dans le répertoire :

http://www.tinyurl.com/yetsofy/GLOBAL05_NOC/2010/SPLIT_FORECAST .

L'ensemble du système est mis en œuvre sur le calculateur CAPARMOR II. En pratique les propriétés de parallélisations du code WWATCH sont largement utilisés car les calculs sont réalisés sur 256 processeurs, avec 3 processeurs dédiés aux entrées-sorties. L' efficacité de la parallélisation est meilleure que 97\%. A titre d'exemple, les 6 domaines emboités ensemble que sont global-nord ouest Europe - Mer du Nord Manche Gascogne - Antilles - Nouvelle Calédonie - Polynésie sont calculés en 30 minutes environ pour 6,5 jours de temps réel (rejeu des dernières $12 \mathrm{~h}$ et prévision à 6 jours). Le domaine de calcul non-structuré sur le Finistère, avec 12000 nœuds de calcul et un pas de temps d'advection de l'ordre de $10 \mathrm{~s}$ prend lui 11 minutes, sur 256 processeurs également. Des tests comparatifs préliminaires avec le code WWM de ROLAND et al. (2009) semblent indiquer que WWATCH serait plusieurs fois plus lent que WWM. Il est probable que l'optimisation des schémas réalisées par A. Roland puisse ainsi faire gagner un temps de calcul précieux. C'est l'objet d'une collaboration entre le SHOM, Météo-France, l'Université de Darmstadt et l'Ifremer. Toutefois, dans le cadre de Prévimer, le facteur limitant sur le temps calcul est désormais la durée du posttraitement, qui se fait de façon séquentielle. L'écriture directe des résultats au format NetCDF devrait réduire ce temps d'au moins un facteur deux. 
Tableau 1. Liste des paramètres calculés par les différents modèles. Il convient de noter que le site Prévimer propose des visualisations de $u b s=1.4 u b r$, et abs $=1.4 \mathrm{abr}$, les valeurs significatives de l'amplitude de l'agitation sur le fond.

\begin{tabular}{ll}
\hline $\boldsymbol{a b b r e ́ v i a t i o n}$ & Nom complet \\
\hline$H s$ & Hauteur significative des vagues $($ Hm0) \\
uss & Vecteur dérive de Stokes en surface \\
$m s s$ & Moyenne du carré des pentes: composantes $x$ et y. \\
$t$ & Période moyenne Tm0,-1 \\
$p h s$ & Hauteur significative de la mer de vent ou houle $n^{\circ} i(i<6)$ \\
$p t h$ & Direction moyenne de la mer de vent ou houle $n^{\circ} i(i<6)$ \\
$p s i$ & Etalement directionnel de la mer de vent ou houle $n^{\circ} i(i<6)$ \\
$p t p$ & Période du pic de la mer de vent ou houle $n^{\circ} i(i<6)$ \\
$d i r$ & Direction moyenne de l'ensemble de l'état de mer \\
$f p$ & Fréquence du pic \\
$d p$ & Direction moyenne à la fréquence pic \\
$f c$ & Fréquence de coupure du modèle pour la résolution explicite de \\
& l'évolution du spectre (au delà une "queue" en $f^{5}$ est appliquée) \\
$t w o$ & Flux de quantité de mouvement des vagues vers les courants \\
$t u s$ & Transport de volume induit par la dérive de Stokes \\
$s p r$ & Etalement directionnel de l'ensemble de l'état de mer \\
$f o c$ & Flux d'énergie par unité de surface entre vagues et circulation \\
$t 02$ & Période moyenne Tm02 \\
$C g E$ & Flux d'énergie intégré sur toutes les directions \\
$u b r$ & Amplitude rms de la vitesse orbitale au fond \\
$a b r$ & Amplitude rms des déplacement induits sur le fond \\
$S x y$ & Tensions de radiation $(3$ composantes: Sxx, Syy, Sxy) \\
\hline &
\end{tabular}

Le système actuel ne propose pas de garantie de service. Malgré quelques soucis de connexion, le site est régulièrement mise à jour de façon nominale. L'objectif est de servir des usages pour lesquels la sécurité des biens et des personnes n'est pas en jeu.

\section{Validation des différents paramètres}

Une des principales avancées permise par l'utilisation de nouveaux paramétrages du déferlement (ARDHUIN et al., 2009 ; ARDHUIN et al., sous presse) est l'accès aux paramètres qui dépendent fortement de la partie haute fréquence du spectre. Ainsi ARDHUIN et al. (2009) ont montré une réduction de l'erreur d'environ 50\% pour la dérive de Stokes en surface, par rapport au même modèle utilisant le paramétrage de BIDLOT et al. (2005). De la même manière, pour une vitesse de vent fixe, le nouveau paramétrage est capable de restituer une grande partie de la sensibilité à l'état de la mer 
de la moyenne des pentes au carré (mss) de la surface. Cette propriété sera exploitée pour le traitement de données de télédétection, telle que l'estimation de la salinité de surface à partir de la mesure radiométrique faite par le nouveau satellite SMOS de l'Agence Spatiale Européenne, lancé en décembre 2009. En effet la température de brillance, dont est déduite la salinité, est sensible aux pentes et à la couverture d'écume.

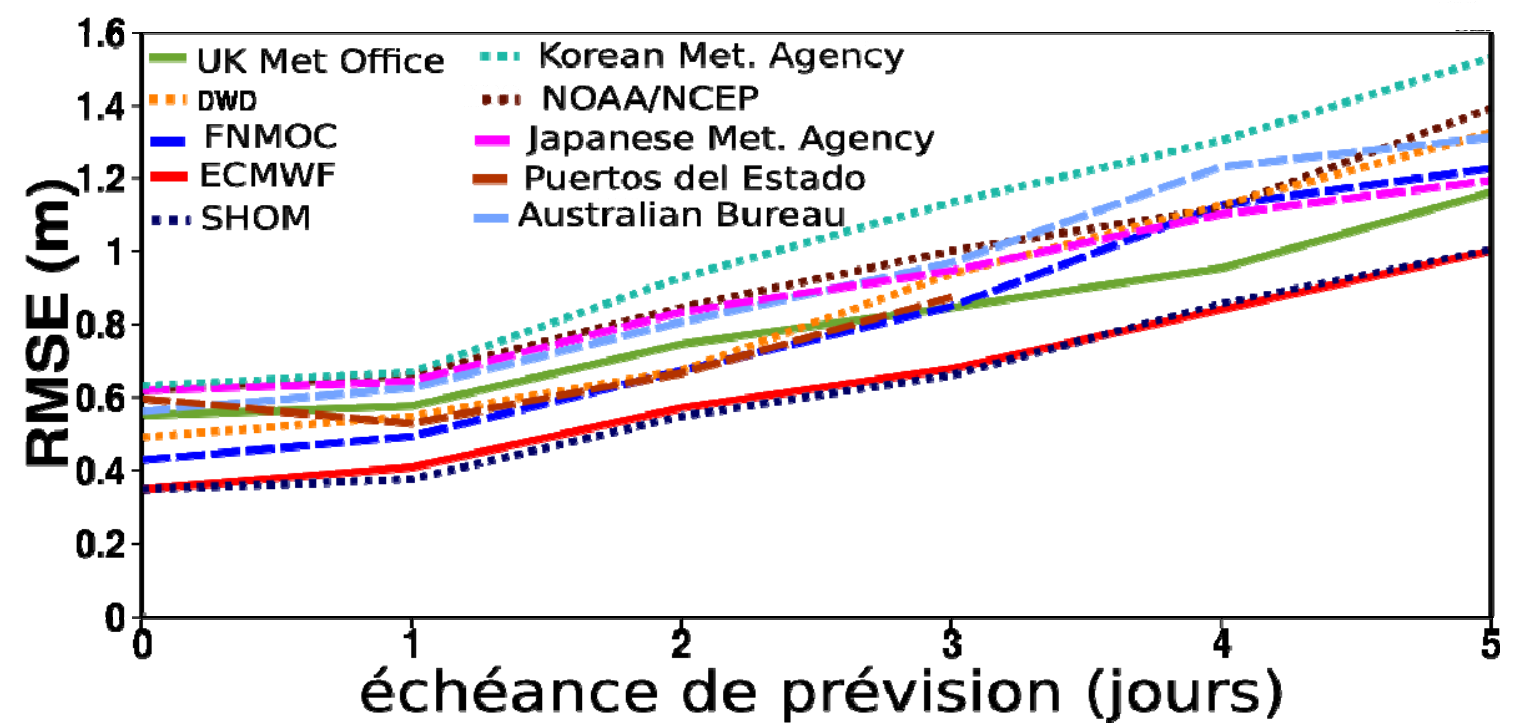

Figure 2. Vérification des différents modèles de prévision dans le cadre du Joint Commission on Marine Meteorology and Oceanography (JCOMM). Figure tirée de BIDLOT (2010). Les statistiques sont calculées pour 10 bouées de l'Atlantique NordEst, pour la période décembre 2009-février 2010. Le modèle SHOM-Ifremer est le modèle qui présente les erreurs les plus faibles, suivi par ECMWF

(RMSE : erreur quadratique moyenne de la prévision).

Pour des applications plus proches du génie côtier, on peut noter une nette amélioration des paramètres d'état de mer au large, comme le montre la figure 3. Un suivi des performances du modèle au large est assuré dans le cadre du JCOMM, et les rapports de validation sont disponible sur leur site web (Par exemple BIDLOT, 2008).

Sur les côtes du Finistère, l'amélioration est encore plus spectaculaire grâce à la prise en compte des courants côtiers. Ainsi, sur la période janvier-mars 2008, l'erreur rms normalisée pour la hauteur significative est passée de 20,5 à 12,2\%, lorsque l'on compare les sorties du modèle heure par heure aux observations.

Cette nette amélioration vient d'une réduction du biais positif dans l'ancien modèle, très visible sur la figure 3. Ainsi, les courants ont tendance à réduire la hauteur des vagues. Cet effet peut être en partie attribué à l'asymétrie des courants, plus forts dans le sens du flot (qui est proche de la direction moyenne des vagues) que dans le sens du jusant, mais il est aussi causé par la réfraction des vagues par les courants. On observe toutefois que 
la modulation du courant dans le modèle, les 8 et 9 mars 2008 par exemple (figure 3 ), est plus faible que dans les observations. Les raisons de cette différence sont en cours d'étude. D'un point de vue quantitatif, la qualité des modélisations est remarquable, proche des résultats au large (MAGNE \& ARDHUIN, 2008). En prenant la moyenne des spectres mesurés sur 3 heures, l'erreur pour le Hs atteint 9.9\%, 11.7\% pour la fréquence moyenne $\mathrm{f}_{\mathrm{m} 0,2}$, et $8.4 \%$ pour la fréquence moyenne $\mathrm{f}_{\mathrm{m} 0,-1}$, contre $17 \%, 14 \%$ et $9.6 \%$ pour le modèle sans courant.
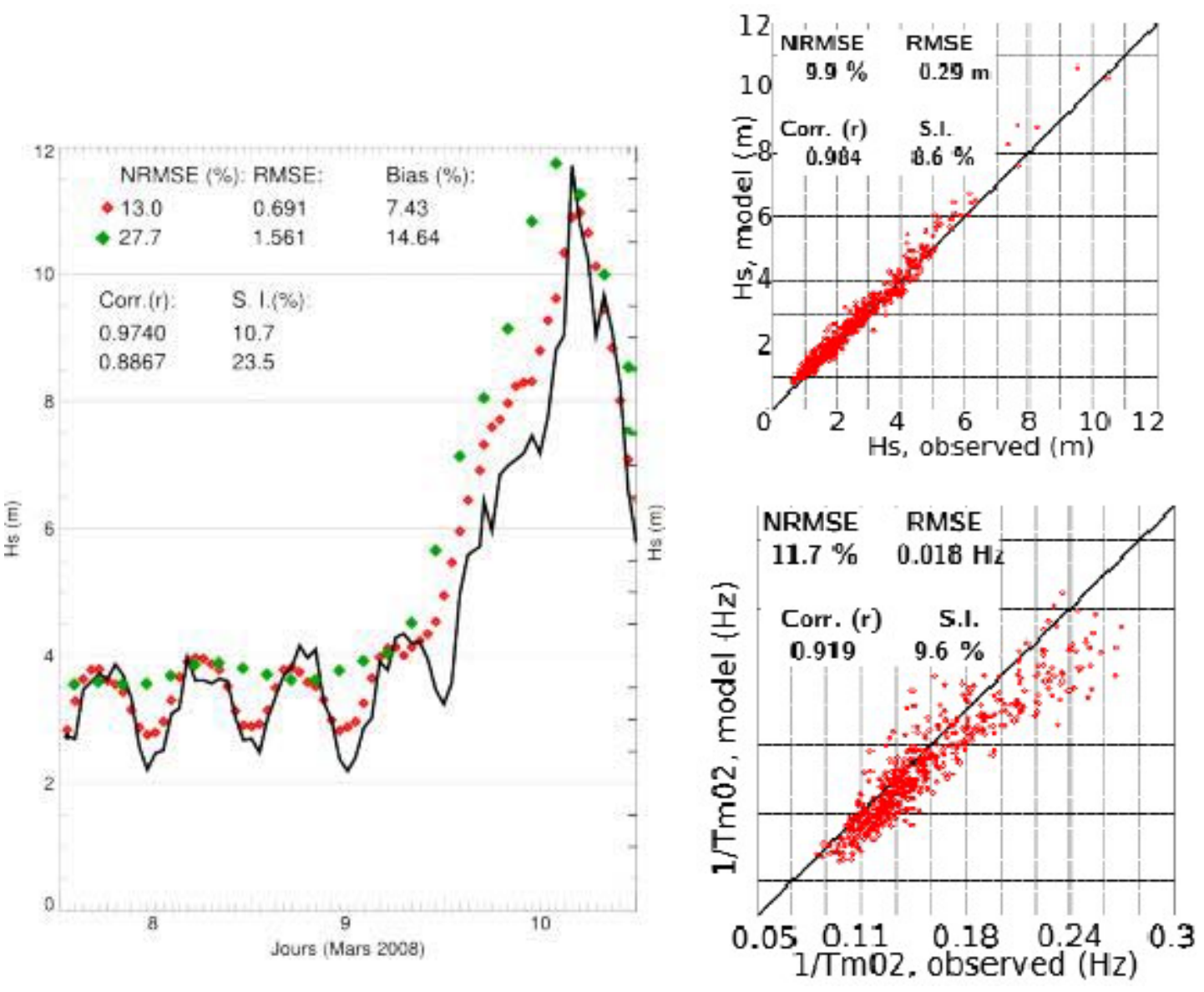

Figure 3. A gauche: comparaison entre Hs mesuré par la bouée des Pierres noires (courbe noire) et les modélisations avec (petits symboles rouges) et sans (gros symboles verts) prise en compte des courants. A droite: comparaison modèle-mesure avec des mesures moyennées sur 3 h, pour la hauteur des vagues (en haut) et la fréquence moyenne (en bas), de janvier à mars 2008.

Cette précision accrue du modèle permet ainsi de s'intéresser à d'autres paramètres, tels que l'indice de hauteur d'eau de HUNT (1959) que l'on peut redéfinir par $\mathrm{H}_{\mathrm{n}}=\mathrm{T}_{\mathrm{m} 0,-1}$ $\left(\mathrm{gH}_{\mathrm{s}}\right)^{0.5}$. Ce paramètre est généralement corrélé aux hauteurs d'ondes infragravitaires, et ainsi aux hauteur des seiches dans les ports, mais aussi au run-up à la côte. $H_{n}$ peut ainsi 
être utilisé comme indice du risque de submersion. Les mesures de pression réalisées en haut de la falaise de l'île de Banneg (voir figure 1), dans le cadre de la campagne SHOM "VIGICOTE 2008", sont fortement corrélées ( $\mathrm{r}=0.94)$ au paramètres $\mathrm{H}_{\mathrm{n}}$ estimé à $1 \mathrm{~km}$ au large de Banneg (figure 4).

Cette forte corrélation entre modèle et observation des niveaux extrêmes permet d'envisager des applications pour l'étude et la cartographie du risque de submersion marine. Idéalement on souhaiterait remplacer le facteur empirique entre le niveau et $\mathrm{H}_{\mathrm{n}}$ ( 0.0743 dans le cas de Banneg) par un modèle déterministe qui prend en compte le profil topo-bathymétrique de l'estran.

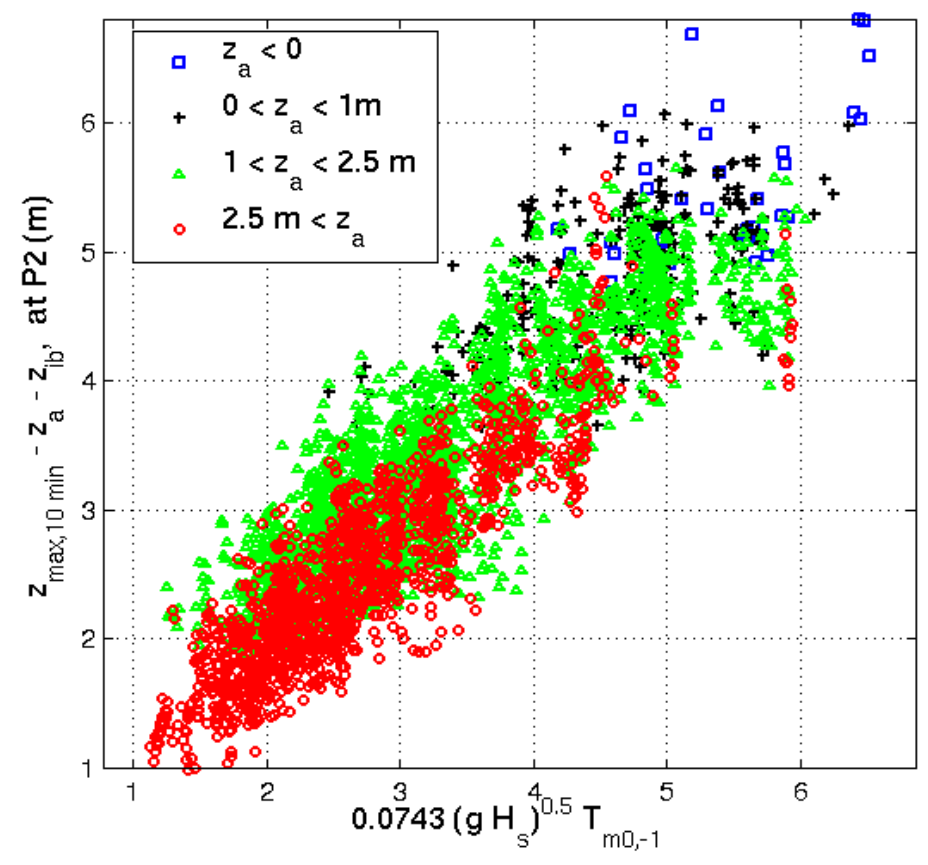

Figure 4. Pression maximale enregistrée par le capteur P2 situé $4.15 \mathrm{~m}$ au dessus du niveau IGN69, soit au dessus du niveau des plus hautes mers astronomiques, comparé à $0.0743 H_{n}$. La pression atmosphérique, la marée astronomique et l'effet de baromètre inverse ont été retranchés. Les mesures toutes les heures entre octobre 2008 et mai 2009 ont été classées par niveau de marée. Bleu: sous niveau moyen, rouge: marée supérieure à $2.5 \mathrm{~m}$ au dessus du niveau moyen. On peut noter un décalage des niveaux avec le niveau de la marée.

\section{Conclusions}

Des progrès important dans la modélisation des états de mer ont permis d'améliorer considérablement les paramètres usuels tels que la hauteur significative et la période moyenne fournis par le système Prévimer. La meilleure représentation de l'état de la mer permet désormais de nouvelles applications, en particulier pour l'étude des risques de submersion marine. 


\section{Remerciements}

Le soutien du pôle de calcul pour la Mer et de la région Bretagne ont été essentiel pour la poursuite du projet Prévimer et des travaux présentés ici. Les campagnes de mesures en mer d'Iroise ont été réalisées avec la participation de David Corman et son équipe (SHOM), de Bernard Fichaut et Serge Suanez (IUEM), avec le concours précieux de la Réserve naturelle d'Iroise, et le soutien de l'ANR dans le cadre du projet HEXECO. Lucia Pinea-Guillou (SHOM) a par ailleurs contribué à l'analyse des niveaux d'eau.

\section{Références bibliographiques}

ARDHUIN F., MARIE L., RASCLE N., FORGET P., ROLAND A. (2009). Observation and estimation of Lagrangian, Stokes and Eulerian currents induced by wind and waves at the sea surface. J. Phys. Oceanogr., vol. 39, no. 11, pp. 2820-2838. doi:10.1175/2009JPO4169.1

ARDHUIN F., ROGERS E., BABANIN A., FILIPOT J.-F., MAGNE R., VAN DER WESTHUYSEN A., QUEFFEULOU P., LEFEVRE J.-M., AOUF L., COLLARD F. (sous presse). Semi-empirical dissipation source functions for wind-wave models: part $i$, definition, calibration and validation. J. Phys. Oceanogr.

BIDLOT J., ABDALLA S., JANSSEN P. (2005). A revised formulation for ocean wave dissipation in CY25R1, Tech. Rep. Memorandum R60.9/JB/0516, Research Department, ECMWF, Reading, U.K.

BIDLOT J.-R. (2010). Intercomparison of operational wave forecasting systems against buoys:data from ECMWF, MetOffice, FNMOC, MSC, NCEP, MeteoFrance, DWD, BoM, SHOM, JMA, KMA, Puerto del Estado, DMI, Joint WMO-IOC Technical Commission for Oceanography and Marine Meteorology. Disponible en ligne sur :

URL http://www.tinyurl.com/7bz6jj

HUNT I.A. (1959). Design of seawalls and breakwaters. Journal of Waterways and Harbours Division, ASCE 85 (WW3), pp 123-152.

MAGNE R., ARDHUIN F. (2008). Prévisions et rejeux des états de mer du globe à la plage. $\mathrm{X}^{\text {èmes }}$ JNGCGC, Sophia Antipolis, pp 375-384. doi: 10.5150/jngcgc.2008.036-M

ROLAND A., CUCCO A., FERRARIN C., HSU T.-W., LIAU J.-M., OU S.-H., UMGEISSER G., ZANKE U. (2009). On the development and verification of a 2-d coupled wave-current model on unstructured meshes, J. Mar. Sys., vol. 78, pp S244S254. doi:10.1016/j.jmarsys.2009.01.026 